University of New Hampshire

University of New Hampshire Scholars' Repository

Physics Scholarship

Physics

$1-27-2009$

\title{
Scale-dependent angle of alignment between velocity and magnetic field fluctuations in solar wind turbulence
}

\author{
J. J. Podesta \\ University of New Hampshire \\ Benjamin D.G. Chandran \\ University of New Hampshire, benjamin.chandran@unh.edu
}

A. Bhattacharjee

University of New Hampshire

D. A. Roberts

NASA

M. L. Goldstein

Follow this and additional works at: https://scholars.unh.edu/physics_facpub

Part of the Physics Commons

\section{Recommended Citation}

Podesta, J. J.; Chandran, Benjamin D.G.; Bhattacharjee, A.; Roberts, D. A.; and Goldstein, M. L., "Scaledependent angle of alignment between velocity and magnetic field fluctuations in solar wind turbulence" (2009). Journal of Geophysical Research-Space Physics. 79.

https://scholars.unh.edu/physics_facpub/79

This Article is brought to you for free and open access by the Physics at University of New Hampshire Scholars' Repository. It has been accepted for inclusion in Physics Scholarship by an authorized administrator of University of New Hampshire Scholars' Repository. For more information, please contact Scholarly.Communication@unh.edu. 


\title{
Scale-dependent angle of alignment between velocity and magnetic field fluctuations in solar wind turbulence
}

\author{
J. J. Podesta, ${ }^{1}$ B. D. G. Chandran, ${ }^{1}$ A. Bhattacharjee, ${ }^{1}$ D. A. Roberts, ${ }^{2}$ \\ and M. L. Goldstein ${ }^{2}$ \\ Received 17 June 2008; revised 30 September 2008; accepted 7 October 2008; published 27 January 2009.
}

[1] Under certain conditions, freely decaying magnetohydrodynamic (MHD) turbulence evolves in such a way that velocity and magnetic field fluctuations $\delta \boldsymbol{v}$ and $\delta \boldsymbol{B}$ approach a state of alignment in which $\delta \boldsymbol{v} \propto \delta \boldsymbol{B}$. This process is called dynamic alignment. Boldyrev has suggested that a similar kind of alignment process occurs as energy cascades from large to small scales through the inertial range in strong incompressible MHD turbulence. In this study, plasma and magnetic field data from the Wind spacecraft, data acquired in the ecliptic plane near $1 \mathrm{AU}$, are employed to investigate the angle $\theta(\tau)$ between velocity and magnetic field fluctuations in the solar wind as a function of the time scale $\tau$ of the fluctuations and to look for the scaling relation $\langle\theta(\tau)\rangle \sim \tau^{1 / 4}$ predicted by Boldyrev. We find that the angle $\langle\theta(\tau)\rangle$ appears to scale like a power law at large inertial range scales, but then deviates from power law behavior at medium to small inertial range scales. We also find that small errors in the velocity vector measurements can lead to large errors in the angle measurements at small time scales. As a result, we cannot rule out the possibility that the observed deviations from power law behavior arise from errors in the velocity measurements. When we fit the data from $2 \times 10^{3} \mathrm{~s}$ to $2 \times 10^{4} \mathrm{~s}$ with a power law of the form $\langle\theta(\tau)\rangle \propto \tau^{p}$, our best fit values for $p$ are in the range $0.27-0.36$.

Citation: Podesta, J. J., B. D. G. Chandran, A. Bhattacharjee, D. A. Roberts, and M. L. Goldstein (2009), Scale-dependent angle of alignment between velocity and magnetic field fluctuations in solar wind turbulence, J. Geophys. Res., 114, A01107, doi:10.1029/2008JA013504.

\section{Introduction}

[2] Incompressible MHD turbulence has been the subject of a large body of theoretical research over the last four decades, beginning with the Iroshnikov-Kraichnan theory [Iroshnikov, 1964; Kraichnan, 1965] which assumed isotropic power spectra. It is now understood that the nonlinear interaction between counter-propagating shear Alfvén wave packets generates an energy cascade mainly in the direction perpendicular to the background magnetic field and produces an anisotropic power spectrum [Montgomery and Turner, 1981; Shebalin et al., 1983; Oughton et al., 1995; Sridhar and Goldreich, 1994; Goldreich and Sridhar, 1995, 1997; Montgomery and Matthaeus, 1995; Ng and Bhattacharjee, 1996; Chen and Kraichnan, 1997; Bhattacharjee and Ng, 2001; Terry et al., 2001; Galtier et al., 2002; Cho and Lazarian, 2003]. The wave number dependence of the anisotropic power spectrum has been calculated by weak and strong turbulence theories as well as by direct numerical simulations [Cho and Vishniac, 2000; Maron and Goldreich,

\footnotetext{
${ }^{1}$ Space Science Center, University of New Hampshire, Durham, New Hampshire, USA.

${ }^{2}$ NASA Goddard Space Flight Center, Laboratory for Solar and Space Physics, Greenbelt, Maryland, USA.

Copyright 2009 by the American Geophysical Union. 0148-0227/09/2008JA013504
}

2001; Ng et al., 2003; Müller et al., 2003]. While there is significant consensus regarding the scaling of the spectrum in the weak-turbulence regime where perturbation theory is valid, the scaling of the spectrum in the strong-turbulence regime where perturbation theory fails is still a matter of debate [Maron and Goldreich, 2001; Ng et al., 2003; Galtier et al., 2005; Boldyrev, 2005, 2006; Beresnyak and Lazarian, 2006, 2007; Lithwick et al., 2007; Mininni and Pouquet, 2007; Chandran, 2008a].

[3] Boldyrev [2005, 2006] has recently proposed a theory of strong incompressible MHD turbulence in a large mean magnetic field $\delta b / B_{0} \ll 1$, where $\delta b$ and $B_{0}$ are the r.m.s. and mean values of the magnetic field. In Boldyrev's theory, the average angle $\theta$ between the velocity and magnetic field fluctuations decreases monotonically as the length scale of the fluctuations decreases, although it is never zero, consistent with a constant energy cascade rate (by Boldyrev's definition, $0 \leq \theta \leq \pi / 2$ ). In particular, Boldyrev predicts that $\langle\theta\rangle \propto \lambda_{\perp}^{1 / 4}$, where $\lambda_{\perp}$ is the scale size of the fluctuations in the plane perpendicular to the local magnetic field and $\langle\cdots\rangle$ represents a time, volume, or ensemble average. This "scale-dependent dynamic alignment" has been observed in direct numerical simulations [Mason et al., 2006, 2008] (but see also Beresnyak and Lazarian [2006]) and causes the energy cascade time to become longer than in the unaligned case. This slowing of the energy cascade becomes increasingly pronounced toward smaller scales. As a result, 
Boldyrev obtains a perpendicular energy spectrum proportional to $k_{\perp}^{-3 / 2}$ instead of the steeper $k_{\perp}^{-5 / 3}$ scaling found in some other studies [e.g., Goldreich and Sridhar, 1995; Cho and Vishniac, 2000]. Simulations by Mason et al. [2006, 2008] also suggest that Boldyrev's alignment effect disappears when the mean magnetic field is not sufficiently strong.

[4] In the solar wind, which is often modeled as an incompressible MHD fluid at large scales, the typical r.m.s. magnetic field fluctuations satisfy $\delta b / B_{0} \lesssim 1$. In the ecliptic plane near the orbit of the Earth at $1 \mathrm{AU}, \delta b / B_{0} \approx$ 0.2 . Therefore, it is possible that Boldyrev's alignment effect may occur in the solar wind. In this paper, we investigate the scale dependence of the average angle $\langle\theta\rangle$ in the solar wind to see whether it follows Boldyrev's theory. We also investigate the more general possibility of the existence of a scaling law $\langle\theta\rangle \propto \lambda_{\perp}^{p}$ within the inertial range for some power law index $p$. Some of the results obtained in the present study, results not discussed here, are discussed by Podesta et al. [2008]. The angle measurements reported by Podesta et al. [2008] are different from those reported here, even though the same data was used in both cases. This is because Podesta et al. [2008] measure the angle using Boldyrev's formula (1) whereas in the present paper the angle is measured using the related formula (8).

[5] The paper is organized as follows. Definitions of the quantities employed in the analysis are presented in section 2. The data preparation and analysis procedures are described in section 3. Measurements of the angle $\theta$ are presented in section 4, and our conclusions are summarized in section 5 .

\section{Quantities Employed in the Analysis}

[6] The formula used by Boldyrev to compute the angle between the fluctuations $\delta \boldsymbol{v}_{\perp}$ and $\delta \boldsymbol{b}_{\perp}$ is [Mason et al., 2006, 2008]

$$
\theta(r)=\sin ^{-1}\left(\frac{\left\langle\left|\delta \boldsymbol{v}_{\perp} \times \delta \boldsymbol{b}_{\perp}\right|\right\rangle}{\left\langle\left|\delta \boldsymbol{v}_{\perp}\right| \cdot\left|\delta \boldsymbol{b}_{\perp}\right|\right\rangle}\right)
$$

where $\boldsymbol{r}$ denotes the spatial separation of the two-point measurement, $r=|\boldsymbol{r}|$ is the length scale of the fluctuations, " $\times$ " denotes the vector cross product, the vectors $\delta \boldsymbol{v}_{\perp}$ and $\delta \boldsymbol{b}_{\perp}$ are the projections of the fluctuations onto the plane perpendicular to the local mean magnetic field $\bar{b}$, the separation vector $\boldsymbol{r} \perp \overline{\boldsymbol{b}}$ lies in the field perpendicular plane, and the angle brackets denote a suitable average.

[7] It should be emphasized that Boldyrev's theory is formulated for velocity fluctuations $\delta v=v(x+r)-v(x)$ and magnetic field fluctuations in which the separation vector $\boldsymbol{r}$ lies in the plane perpendicular to the local mean magnetic field $\bar{b}$. Such separation vectors can be obtained using single spacecraft measurements of solar wind turbulence, but only occasionally and when the mean magnetic field is perpendicular to the average flow velocity.

[8] Recall that single spacecraft measurements are performed along a radial line $\boldsymbol{r}=\boldsymbol{V}_{\mathrm{sw}} \tau$ formed by the rapid advection of the wind past the spacecraft (Taylor's frozen flow hypothesis). The direction of the mean magnetic field is often aligned with the Parker spiral which, in the ecliptic plane at $1 \mathrm{AU}$, is inclined at approximately 45 degrees to the radial direction. Thus, solar wind fluctuations are measured with separation vectors typically near 45 degrees to the mean magnetic field. The questions is: Can velocity and magnetic field fluctuations measured in this way provide a meaningful test of Boldyrev's scaling law? The answer is yes, provided solar wind turbulence is dominated by so called quasi-2-D fluctuations in which the variations in directions perpendicular to $\bar{b}$ are much greater than the variations parallel to $\bar{b}$. In this case, the contribution to $\delta \boldsymbol{v}=$ $\boldsymbol{v}(\boldsymbol{x}+\boldsymbol{r})-\boldsymbol{v}(\boldsymbol{x})$ arising from the component of $\boldsymbol{r}=\boldsymbol{V}_{\mathrm{sw}} \tau$ along $\bar{b}$ can be neglected. In other words, for the quasi-2-D component of the turbulence, the velocity and magnetic field fluctuations along the displacement $\boldsymbol{r}=\boldsymbol{V}_{\mathrm{sw}} \tau$ are approximately equal to the velocity and magnetic field fluctuations along $\boldsymbol{r}_{\perp}=\boldsymbol{r}-(\boldsymbol{r} \cdot \hat{\boldsymbol{u}}) \hat{\boldsymbol{u}}$, where $\hat{\boldsymbol{u}}$ is the unit vector in the direction of $\overline{\boldsymbol{b}}$.

[9] How often the solar wind is dominated by quasi-2-D fluctuations is an important question that is not completely settled. Recent work by Dasso et al. [2005] has suggested that the turbulence in low-speed wind is predominantly quasi-2-D while that in high-speed wind is not. Here we analyze both high- and low-speed wind.

[10] For single-spacecraft solar wind data, fluctuations of scale size $\tau$ are defined by

$$
\begin{gathered}
\delta \boldsymbol{v}(t, \tau)=\boldsymbol{v}(t+\tau)-\boldsymbol{v}(t), \\
\delta \boldsymbol{b}(t, \tau)=\boldsymbol{b}(t+\tau)-\boldsymbol{b}(t),
\end{gathered}
$$

where $\boldsymbol{v}(t)$ is the solar wind velocity vector (proton velocity) at time $t, \boldsymbol{b}(t)=\boldsymbol{B}(t) /\left(\mu_{0} \rho(t)\right)^{1 / 2}$ is the magnetic field in velocity units, $\mu_{0}$ is the permeability of free space (SI units), $\rho(t)=1.16 m_{p} n_{p}(t)$ is the approximate mass density of the solar wind, $m_{p}$ is the proton mass, $n_{p}(t)$ is the proton number density, and the factor 1.16 accounts approximately for the mass of the alpha particles. All quantities are measured in the spacecraft frame of reference. The time scale $\tau$ is equivalent to a length scale $r=V_{\text {sw }} \tau$ along the mean flow direction.

[11] The perpendicular projections of the fluctuations are defined by

$$
\begin{aligned}
& \delta \boldsymbol{v}_{\perp}=\delta \boldsymbol{v}-(\delta \boldsymbol{v} \cdot \hat{\boldsymbol{u}}) \hat{\boldsymbol{u}} \\
& \delta \boldsymbol{b}_{\perp}=\delta \boldsymbol{b}-(\delta \boldsymbol{b} \cdot \hat{\boldsymbol{u}}) \hat{\boldsymbol{u}}
\end{aligned}
$$

where $\hat{\boldsymbol{u}}=\overline{\boldsymbol{b}} /|\overline{\boldsymbol{b}}|$ is the unit vector in the direction of the local mean magnetic field $\bar{b}$. These are the quantities employed in equation (1).

[12] The scale size of the local mean magnetic field $\bar{b}$ is typically chosen to be an order of magnitude greater than that of the fluctuations themselves. This provides a separation of scales consistent with the notion that the local mean field acts as a guide field for the fluctuations. For fluctuations of scale size $\tau$, the averaging window used to measure the mean magnetic field in this study is of length $3 \tau$. Hence, the mean magnetic field $\overline{\boldsymbol{b}}(t, \tau)$ is measured over the time interval from $t-\tau$ to $t+2 \tau$ and, as a consequence, $\overline{\boldsymbol{b}}(t, \tau)$ is a function of both the time $t$ at which the fluctuations are measured and the scale size $\tau$ of the fluctuations. Loosely 


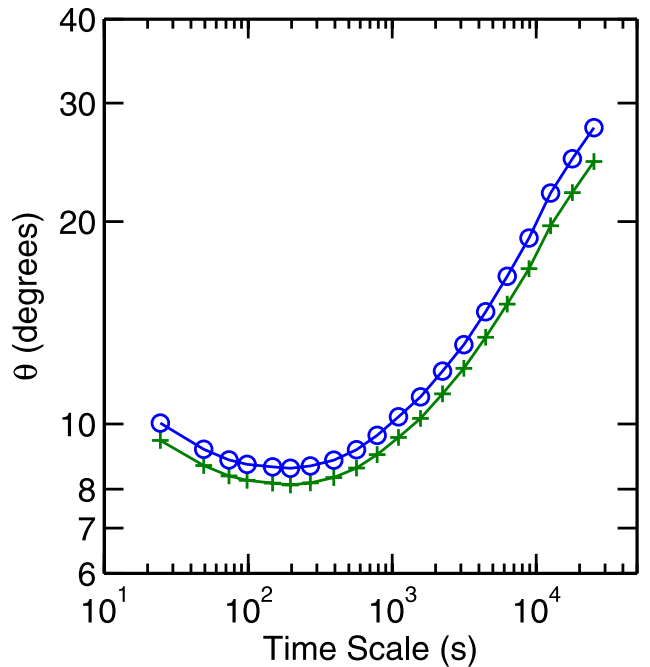

Figure 1. Comparison between the average angle of alignment computed using Boldyrev's formula (1) (plus symbols) and the prescription (7) and (8) (circles).

speaking, it is the average magnetic field "felt" by the fluctuations.

[13] Note that equation (1) defines an average angle, in some sense. In practice, it is also of interest to examine the distribution of the angle $\theta$. To achieve this, Boldyrev's definition (1) may be generalized as follows. Note that the formula (1) weighs the contributions to the "average angle" by the amplitude of the fluctuations so that fluctuations with larger values of $\left|\delta \boldsymbol{v}_{\perp}\right| \cdot\left|\delta \boldsymbol{b}_{\perp}\right|$ contribute more to the average. Thus, for each pair of fluctuations $\left(\delta \boldsymbol{v}_{\perp}, \delta \boldsymbol{b}_{\perp}\right)$ in the data list one can define a weight

$$
w_{n} \propto\left|\delta \boldsymbol{v}_{\perp}\right| \cdot\left|\delta \boldsymbol{b}_{\perp}\right|, \quad \sum_{n=1}^{N} w_{n}=1,
$$

and an angle

$$
\theta_{n}=\sin ^{-1}\left(\frac{\left|\delta \boldsymbol{v}_{\perp} \times \delta \boldsymbol{b}_{\perp}\right|}{\left|\delta \boldsymbol{v}_{\perp}\right| \cdot\left|\delta \boldsymbol{b}_{\perp}\right|}\right)
$$

where $0 \leq \sin ^{-1}(x)<\pi / 2$. Then, the average angle is defined by

$$
\langle\theta\rangle=\sum_{n=1}^{N} w_{n} \theta_{n}
$$

and the variance of the angle is defined by

$$
\left\langle(\theta-\bar{\theta})^{2}\right\rangle=\sum_{n=1}^{N} w_{n}\left(\theta_{n}-\bar{\theta}\right)^{2},
$$

where $\bar{\theta}=\langle\theta\rangle$. The empirically determined probability distribution for the angle $\theta$ is defined by the relation

$$
\operatorname{Prob}\{\alpha<\theta<\beta\}=\sum_{\alpha<\theta_{n}<\beta} w_{n},
$$

where the sum includes all values of $n$ such that $\alpha<\theta_{n}<\beta$.

[14] For the purposes of this study, the average angle computed using equations (1) and (8) are roughly equal for all the cases encountered here. A typical example of the angles computed using equations (1) and (8) is shown in
Figure 1. It should be noted that other choices of weights are possible, such as $w_{n}=|\delta \boldsymbol{v}|^{2}+|\delta \boldsymbol{b}|^{2}$, for example. These possibilities shall not be considered further.

[15] Note that the formula (1) imposes the restriction $0 \leq$ $\theta \leq \pi / 2$ and does not make any distinction between the angles $\theta$ and $\pi-\theta$. In other words, the angles $\theta$ and $\pi-\theta$ are equivalent in the formulation (1). Alternatively, it is possible to study the range of angles $0 \leq \theta \leq \pi$ as in the work by Matthaeus et al. [2008]. This is useful because, for Alfvén waves, alignment angles near $\pi$ are associated with fluctuations propagating in the direction of the mean magnetic field $\left(\boldsymbol{k} \cdot \boldsymbol{B}_{0}>0\right)$ and angles near 0 are associated with fluctuations propagating in the opposite direction $\left(\boldsymbol{k} \cdot \boldsymbol{B}_{0}<0\right)$. This approach allows populations of forward and backward propagating fluctuations to be studied separately. For implementation, it is only necessary to replace the angle (7) with the definition

$$
\theta_{n}=\cos ^{-1}\left(\frac{\delta \boldsymbol{v}_{\perp} \cdot \delta \boldsymbol{b}_{\perp}}{\left|\delta \boldsymbol{v}_{\perp}\right|\left|\delta \boldsymbol{b}_{\perp}\right|}\right)
$$

where $0 \leq \cos ^{-1}(x) \leq \pi$. The weights remain the same. Two probabilities of interest are the probabilities that the fluctuations are propagating in the direction of the mean magnetic field or opposite to the direction of the mean magnetic field, namely, $\operatorname{Prob}(\pi / 2<\theta<\pi)$ and $\operatorname{Prob}(0<\theta<$ $\pi / 2$ ), respectively.

\section{Data Preparation and Analysis Procedures}

[16] The data employed in this study consist of simultaneous measurements of the proton velocity vector $\boldsymbol{v}$ and magnetic field vector $\boldsymbol{B}$ obtained by the Wind threedimensional plasma (3DP) instrument [Lin et al., 1995] and the Wind magnetic field instrument [Lepping et al., 1995], respectively. The merged plasma and magnetic field data, commonly called PLSP data, has a typical sampling time of $\sim 24 \mathrm{~s}$ which changes slowly owing to small variations in the spin rate of the spacecraft. In addition, the sampling time sometimes changes to $\sim 12 \mathrm{~s}$ (burst mode) and sometimes to $\sim 48$ s. There are also data gaps of varying size.

[17] For each of the time intervals analyzed in this study Wind was outside the influences of the Earth's magnetosphere and bow shock. The characteristics of the solar wind for each of the time intervals analyzed are summarized in Table 1. The data for each time interval was manually inspected for outliers and irregular behavior. The number of outliers and suspicious data deleted during the inspection process amounts to much less than one percent of the data. Before the data was inspected for outliers the ordering of the time stamps on the data were checked. Occasionally, but rarely, negative time increments were found between successive data records and either the time stamps were corrected where it seemed appropriate, or the data were reordered in time or deleted.

[18] For each time interval, the analysis procedure consists of stepping sequentially through the data list and computing the fluctuations $\delta \boldsymbol{v}$ and $\delta \boldsymbol{b}$ at each time step. At each time $t$ one computes the fluctuations $\delta \boldsymbol{v}$ and $\delta \boldsymbol{b}$ using equations (2) and (3), the mean magnetic field $\overline{\boldsymbol{b}}$ by averaging the magnetic field vector $\boldsymbol{b}$ over the interval $t-\tau$ to $t+2 \tau$, and the projections of the fluctuations onto 
Table 1. Time Intervals Employed in the Analysis ${ }^{\mathrm{a}}$

\begin{tabular}{|c|c|c|c|c|c|c|c|c|}
\hline Interval & Begin & End & Days & Description & $\left|\bar{v}_{x}\right|$ & $\sigma_{v_{x}}$ & $\bar{n}_{p}$ & $\sigma_{n_{p}}$ \\
\hline 1 & 01 Jan 1995 & 29 Jul 1995 & 209 & before minimum; quasi-periodic HS streams & 457 & 125 & 6.5 & 4.7 \\
\hline 2 & 15 May 1996 & 16 Aug 1996 & 93 & solar minimum; predominantly LS wind & 398 & 52 & 9.0 & 4.9 \\
\hline 3 & 08 Jan 1997 & 09 Jun 1997 & 152 & after minimum; LS wind; some streams & 403 & 75 & 9.1 & 6.0 \\
\hline 4 & 23 Aug 2000 & 15 Feb 2001 & 176 & solar max; variable wind; some HS streams & 430 & 100 & 7.0 & 6.0 \\
\hline
\end{tabular}

${ }^{\mathrm{a}}$ The units of proton velocity $v_{x}$ and density $n_{p}$ are $\mathrm{km} / \mathrm{s}$ and particles per cubic centimeter, respectively. The rms values are defined by $\sigma_{v_{x}}{ }^{2}=\left\langle\left(v_{x}-\bar{v}_{x}\right)^{2}\right\rangle$, where $\bar{v}_{x}$ is the sample mean (GSE coordinates) and the angle brackets denote a time average over the given interval. HS, high-speed; LS, low-speed.

the plane perpendicular to $\overline{\boldsymbol{b}}$ using equations (4) and (5). By stepping sequentially through the list, one obtains the average alignment angle using either Boldyrev's formula (1) or the approximately equivalent formula (8). This procedure is then repeated for each time scale $\tau$ of interest. Because the data are not uniformly spaced in time it is necessary to introduce an error tolerance $e$ so that a time interval of length $\sim \tau$ is accepted if its length is within the range $(1 \pm e) \tau$. Typically, tolerances around 1 percent are used. For a given value of $\tau$ the tolerance $e$ is constant, but the value of $e$ may change for different values of $\tau$. In particular, larger tolerances are employed at the smallest time scales to ensure a uniform size of the statistical sample at all time scales.

[19] For completeness, it should be mentioned that the number of statistical samples used to compute the average angle for intervals 1 through 4 in Table 1 are approximately $6 \times 10^{5}, 2.8 \times 10^{5}, 4.3 \times 10^{5}$, and $5.8 \times 10^{5}$, respectively.

\section{Measurements of the Angle $\theta$ 4.1. Probability Distributions}

[20] The probability density functions (PDFs) for the angles measured using equation (11) are shown in Figure 2. The PDFs in Figure 2 show that in solar wind turbulence the fluctuations $\delta \boldsymbol{v}_{\perp}(\tau)$ and $\delta \boldsymbol{b}_{\perp}(\tau)$ become progressively more aligned as $\tau$ decreases from $\sim 2 \times 10^{4}$ to a few hundred seconds. A related behavior is observed in simulations of incompressible MHD turbulence where alignment between $\boldsymbol{v}$ and $\boldsymbol{b}$ is observed to occur locally in patches and on a rapid time scale that is less than the eddy turnover time [Matthaeus et al., 2008].

[21] In the ecliptic plane, the cross-helicity of solar wind turbulence alternates in sign from one magnetic sector to the next so one would expect the cross helicity to approach zero over long averaging times (one or more solar rotations). This should produce equal peaks near 0 and 180 degrees as is found in the bottom right-hand plot in Figure 2 for data near solar maximum in 2000/2001. In the other plots, the peak values near 0 and 180 degrees are not equal indicating that the average cross-helicity in these intervals has not averaged to zero. This may be due to variations in the characteristics of the turbulence caused, for example, by mixing of high- and low-speed wind over long time periods.

[22] Note that the quantity $\langle\cos \theta\rangle$ is roughly equal to the normalized cross-helicity of the turbulence if $\left\langle|\delta \boldsymbol{v}|^{2}\right\rangle \simeq$ $\left\langle|\delta \boldsymbol{b}|^{2}\right\rangle$; in the inertial range this is roughly satisfied since $\left\langle|\delta \boldsymbol{v}|^{2}\right\rangle /\left\langle|\delta \boldsymbol{b}|^{2}\right\rangle \simeq 1 / 2$. Although the cross-helicity and normalized cross-helicity spectrum in the solar wind have been studied for many years [Matthaeus and Goldstein, 1982; Roberts et al., 1987a, 1987b; Tu et al., 1989; Marsch and Tu, 1990; Marsch, 1991; Tu and Marsch, 1995; Milano et al., 2004], $\langle\cos \theta\rangle$ is a signed quantity from which it is not possible to calculate $\langle|\theta|\rangle$.

\subsection{Average Alignment Angle}

[23] Results for the average alignment angle $\theta$ computed using equation (8) are shown in Figure 3 for the four data records listed in Table 1. A linear least squares fit performed on the log-log plot over the decade from $2 \times 10^{3}$ s to $2 \times$ $10^{4} \mathrm{~s}$ yields the best fit power law exponent $p$. Figure 3 shows that the average angle $\langle|\theta(\tau)|\rangle$ decreases as $\tau$ decreases from $\sim 10^{4} \mathrm{~s}$ down to a few times $10^{2} \mathrm{~s}$, and that $\langle|\theta(\tau)|\rangle$ increases as $\tau$ decreases below $\sim 10^{2} \mathrm{~s}$. The inertial range of the turbulence spans time scales from $\sim 10 \mathrm{~s}$ to $\sim 10^{4}$ s (roughly). The failure of any power law to match the data throughout this range would appear at first glance to refute Boldyrev's scaling law. However, there are two considerations that prevent us from concluding that Boldyrev's scaling law is inapplicable to the solar wind.

[24] First, errors in the data can affect our measurements of $\theta$, particularly at small time scales. Suppose the magnetic field data is error free and let $\pm \varepsilon$ be the measurement uncertainty or error associated with each of the velocity components $v_{x}, v_{y}$, and $v_{z}$. Then the error associated with the velocity difference $\delta v_{x}$ is $2 \varepsilon$. If the $z$-axis is taken along the
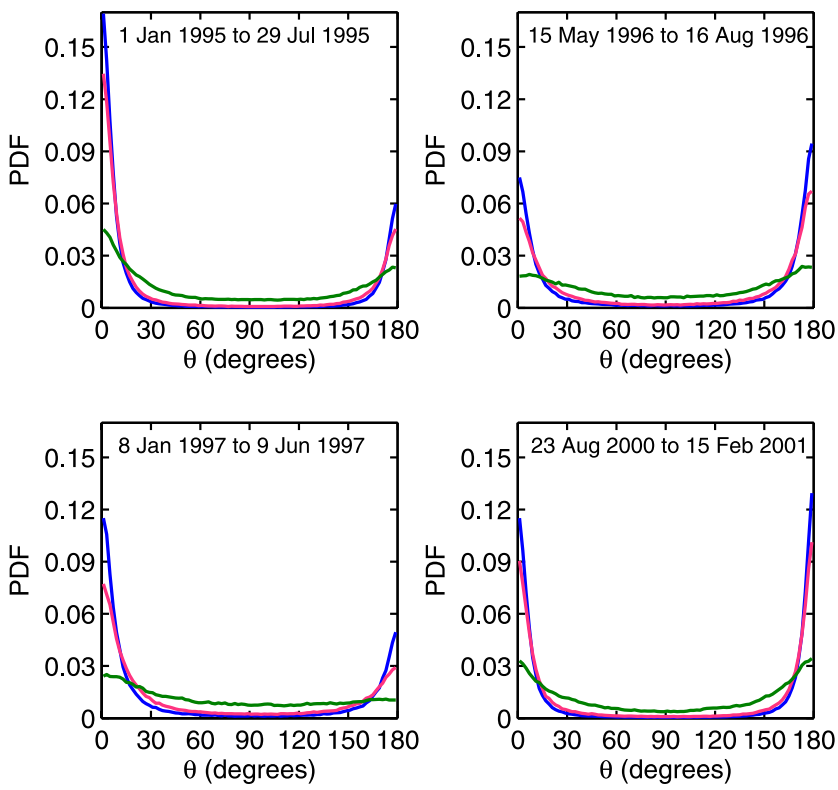

Figure 2. Probability density functions (PDFs) for the angle $\theta$ formed by the vector velocity and magnetic field fluctuations computed from equation (11) at the three time scales $\tau=264,2184$, and 24576 s: blue, pink, and green, respectively. 

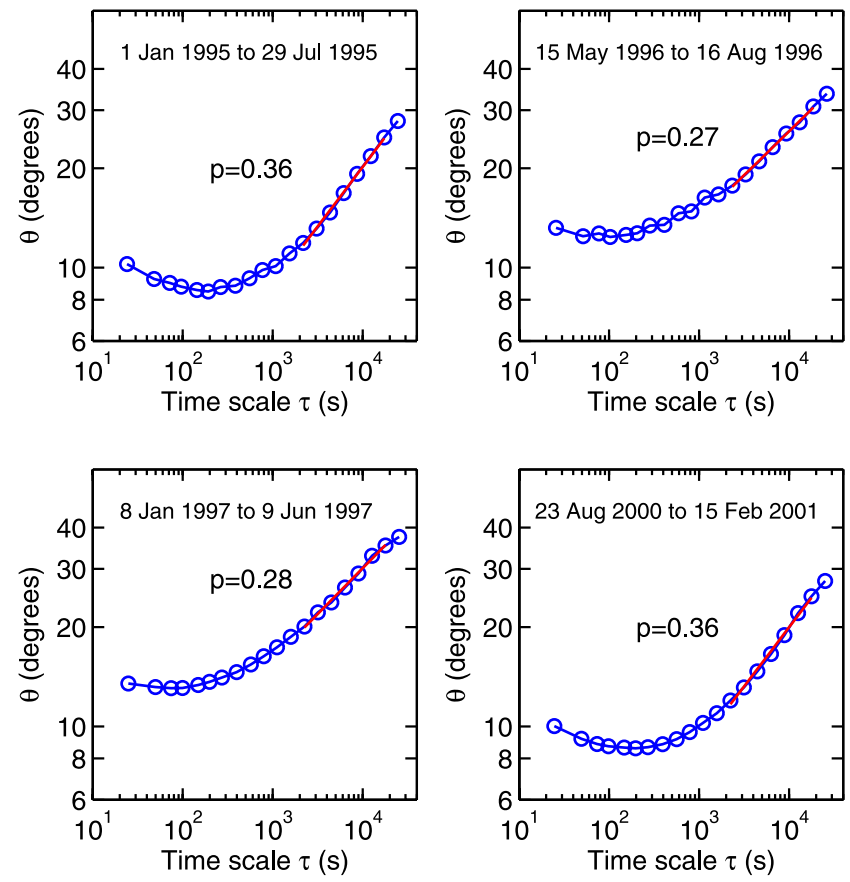

Figure 3. Average angle $\theta$ (blue circles) versus the time scale $\tau$ computed from equation (8) for the four data records listed in Table 1. The red lines indicate a least squares fit with power law exponent $p$ over the range $2 \times 10^{3}$ s to $2 \times$ $10^{4} \mathrm{~s}$. Typically, in the solar wind near $1 \mathrm{AU}$, the inertial range extends from $\sim 10 \mathrm{~s}$ to $\sim 10^{4} \mathrm{~s}$.

direction of the vector $\delta \boldsymbol{b}$, then the angle $\theta$ is approximately given by $\sin \theta \simeq \delta v_{x} /|\delta \boldsymbol{v}|$. If $\delta v_{x}$ is in error by $2 \varepsilon$, then replacing $\delta v_{x}$ with $\delta v_{x}+2 \varepsilon$ and $\theta$ with $\theta+\Delta \theta$, one obtains $\sin (\theta+\Delta \theta) \simeq\left(\delta v_{x}+2 \varepsilon\right) /|\delta \boldsymbol{v}|$. Hence, assuming $\theta$ and $\Delta \theta$ are both small, the error in the angle $\theta$ is $\Delta \theta \simeq 2 \varepsilon /|\delta \boldsymbol{v}|$. For a typical fluctuation, the quantity $|\delta \boldsymbol{v}|$ is approximately equal to its rms value $\delta v=\left(\left\langle|\delta v|^{2}\right\rangle\right)^{1 / 2}$ so that the error in the angle $\theta$ is $\Delta \theta \simeq 2 \varepsilon / \delta v(\tau)$. This angle $\Delta \theta$ is the smallest alignment angle that can be resolved at time scale $\tau$. We emphasize the important point that $\Delta \theta$ is increasing as $\tau$ decreases because the velocity fluctuations $\delta v(\tau)$ are decreasing. The magnitude of $\Delta \theta$ is uncertain, however, because the measurement error $\varepsilon$ is not known with certainty. Rough estimates suggest it is possible that solar wind fluctuations obey a scaling law of the form $\langle|\theta|\rangle \propto \tau^{p}$ for some $p$ and that measurement errors cause the measured value of $\langle|\theta|\rangle$ to flatten out near $10^{3} \mathrm{~s}$, reach a minimum near $200 \mathrm{~s}$, and then increase toward smaller $\tau$. For the data studied here, we suspect that values of $\varepsilon$ from 0.4 to $0.8 \mathrm{~km} / \mathrm{s}$ would be sufficient to explain both the flattening of the curves near $10^{3} \mathrm{~s}$ and the increase below $200 \mathrm{~s}$.

[25] In addition to measurement errors, a second consideration that prevents us from concluding that Boldyrev's theory is inapplicable to the solar wind is the possibility that the alignment process is spoiled by the presence of compressible fluctuations, for example. Solar-wind turbulence can be viewed as consisting of different components. The energetically dominant component may consist of quasi-2-D incompressible strong Alfvénic turbulence [Bieber et al., 1994, 1996; M. L. Goldstein et al., 1995], which presumably can be accurately modeled as strong incompressible MHD turbulence (see A. A. Schekochihin et al. (Astrophysical gyrokinetics: Kinetic and fluid turbulent cascades in magnetized weakly collisional plasmas, 2007, arXiv:0704.0044) and Howes et al. [2008] for a detailed discussion of this point based on plasma kinetic theory.) It is this "quasi-2-D component" to which Boldyrev's theory would apply. Additional components could include compressible fluctuations or quasi-parallel propagating ("slab") Alfvén waves [see, e.g., Barnes, 1983; Marsch, 1991; Dasso et al., 2005; Chandran, 2005, 2008b]. However, these components are not addressed by Boldyrev [2005, 2006] and are not expected to undergo the same kind of alignment process as in Boldyrev's theory. Denote the amplitude of the velocity fluctuation for the quasi-2-D component of the turbulence by $\delta \boldsymbol{v}_{2 D}(\tau)$ and the amplitude of all the additional components of the turbulence by $\delta \boldsymbol{v}^{\prime}(\tau)$. If, for simplicity, we assume the additional components of the turbulence have no systematic alignment between velocity and magnetic field fluctuations, then the smallest observable value of $\theta$ would be $\theta_{\min } \sim 2 \delta v^{\prime}(\tau) / \delta v_{2 D}(\tau)$. (The reasoning here can be recovered from our discussion of the effects of measurement error above, replacing $\varepsilon$ with $\delta v^{\prime}$.) If the additional components of the turbulence are to some extent aligned, then the smallest observable value of $\theta$ would be smaller. If the additional components of the turbulence possess a power-law velocity spectrum similar to the quasi-2-D component, then $\theta_{\min }$ depends only weakly on $\tau$. If, for

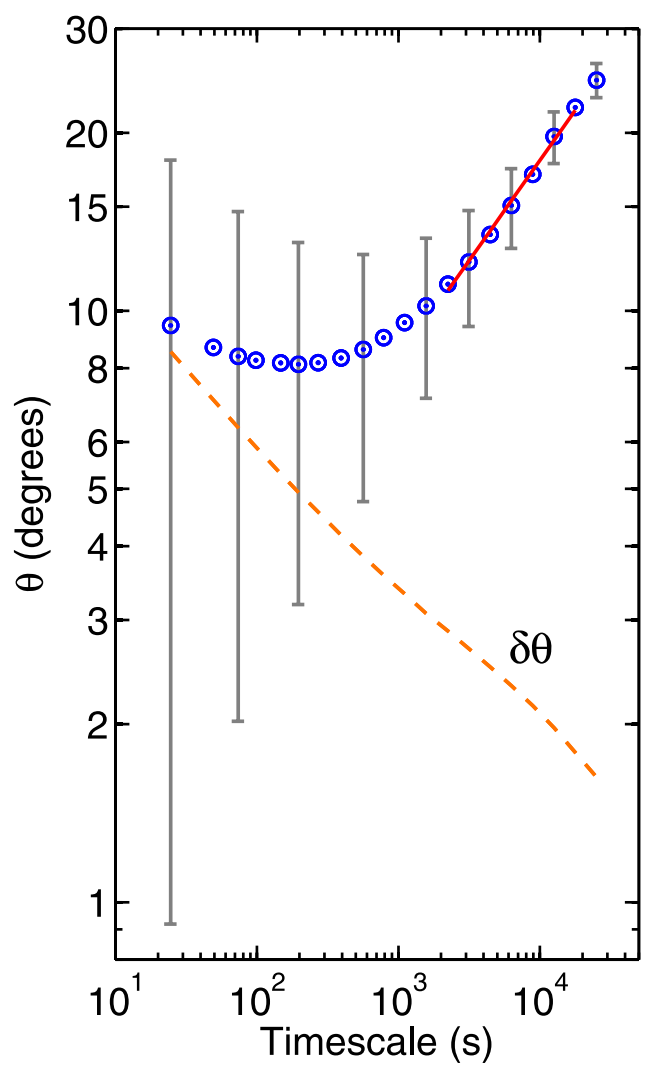

Figure 4. The average angle $\theta$ (circles), the error $\delta \theta=2 \varepsilon /$ $\delta v$ assuming $\varepsilon=0.76 \mathrm{~km} / \mathrm{s}$ (dashed), and the corresponding error bars $\theta \pm \delta \theta$ for interval number 4 in Table 1 . Note that these are not true error bars, but only a rough indication of the smallest resolvable angle at each scale. 
example, $\delta v^{\prime}(\tau) / \delta v_{2 D}(\tau) \simeq 0.1$, then $\theta_{\min }$ would be $\sim 10$ degrees.

[26] In the preceding discussion, we considered the effects of velocity errors and non-quasi-2-D fluctuations separately. A more realistic approach would be to model the data accounting for both effects simultaneously. However, given our lack of knowledge about the magnitude of the velocity error as well as the amplitudes and spectra of the different components of the turbulence, we do not attempt to develop such a model in this paper.

\subsection{Error Bars}

[27] The rough error estimate obtained in section 4.2 can be used to derive rough error bars for the angle measurements in Figure 3. We emphasize that these are not true error bars, only a rough indication of the smallest resolvable angle at each scale. Using the assumed typical value $\varepsilon=$ $0.76 \mathrm{~km} / \mathrm{s}$ and the rms velocity fluctuations $\delta v(\tau)$ computed from the data, we compute the estimated error $\delta \theta=2 \varepsilon / \delta v$. The result for $\delta \theta$ is shown by the dashed orange line in Figure 4. Note that at the smallest scales the magnitude of $\delta \theta$ is comparable to the measured average angle $\theta$. This leads us to believe that the measurements at the smallest scales are unreliable. The error bars in Figure 4 furthermore suggest that much of data in the middle of the range is unreliable too. Only the data at the largest inertial range scales are considered to be sufficiently reliable for the purpose of estimating the power law exponent in the scaling law.

\section{Discussion and Conclusions}

[28] In this paper our goal is to determine whether Boldyrev's recent theory of strong incompressible MHD turbulence [Boldyrev, 2006] is consistent with observations of the angle $\theta(\tau)$ between $\delta \boldsymbol{v}(\tau)$ and $\delta \boldsymbol{b}(\tau)$ in solar wind turbulence. Using data from the Wind spacecraft, we find that $\langle\theta(\tau)\rangle$ decreases as $\tau$ decreases from $\sim 10^{4} \mathrm{~s}$ down to a few times $10^{2} \mathrm{~s}$, and $\langle\theta(\tau)\rangle$ increases as $\tau$ decreases below $\sim 10^{2}$ s. The finding that $d\langle\theta(\tau)\rangle / d \tau<0$ at $\tau \lesssim 10^{2}$ is clearly inconsistent with Boldyrev's prediction that $\langle\theta(\tau)\rangle \propto \tau^{1 / 4}$. However, as discussed in section 4 , the increase in $\langle\theta(\tau)\rangle$ as $\tau$ decreases below $10^{2} \mathrm{~s}$ could result from errors in the velocity measurements. As a result, we cannot conclude from the observations of $\langle\theta(\tau)\rangle$ at small $\tau$ that Boldyrev's theory is inapplicable to the solar wind.

[29] Fits to the average angle over the largest decade of the inertial range where angle measurements are most reliable, $2 \times 10^{3} \mathrm{~s}$ to $2 \times 10^{4} \mathrm{~s}$, suggest that the observed angle obeys a power-law scaling over that decade. Two of the fitted exponents, 0.27 and 0.28 , are close to Boldyrev's theoretical value 0.25 , and the two other measurements, both 0.36 , are significantly different from 0.25 . These disparate findings may be related to the different solar wind conditions found in the four intervals studied. The power law exponents 0.27 and 0.28 were obtained during 1996 and 1997 , periods of mostly slow wind, while the exponents 0.36 and 0.36 were obtained during 1995 and 2000/2001, periods containing a notable presence of high-speed streams. Previous studies have shown that solar wind turbulence is markedly different in high- and low-speed wind and these differences may effect the scaling law for the angle. In light of the study by Dasso et al. [2005] suggesting the turbulence is dominated by quasi-perpendicular fluctuations in low-speed wind and quasi-parallel fluctuations in high-speed wind, it is perhaps not surprising that better agreement with Boldyrev's scaling law is obtained for low-speed wind. More study of this issue is needed.

[30] We note that a more complete test of the applicability of Boldyrev's theory to the solar wind would include an analysis of the power spectrum of solar wind turbulence. Although we have not focused on power spectra in this paper, a number of studies have computed power spectra for solar wind turbulence, finding a range of power-law exponents. Most of the measured exponents are closer to the Kolmogorov value of $5 / 3$ than the value of $3 / 2$ predicted by Boldyrev, and a number are actually larger than $5 / 3$ [Matthaeus and Goldstein, 1982; B. E. Goldstein et al., 1995; Tu and Marsch, 1995; Smith, 2003; Bruno and Carbone, 2005; Podesta et al., 2006, 2007]. Taken together, these results might appear to show that Boldyrev's theory does not apply to the solar wind. However, the use of the spectral index to test Boldyrev's theory is complicated by the fact that Boldyrev's theory is formulated for the case of zero crosshelicity, whereas the cross-helicity is nonzero in the solar wind. A statistical-closure analysis of MHD turbulence with cross helicity that assumes isotropy [Grappin et al., 1983] as well as a recent phenomenological theory of strong anisotropic MHD turbulence with cross helicity [Chandran, 2008a] find that cross helicity steepens the power spectrum relative to the zero-cross-helicity case (but see also Lithwick et al. [2007] and Beresnyak and Lazarian [2007]).

[31] Acknowledgments. The authors thank Stanislav Boldyrev and Davin Larson for helpful discussions and comments. We thank Ronald Lepping, the principal investigator of the Wind magnetic fields investigation (MFI), and Robert Lin, the principal investigator of the Wind threedimensional plasma instrument (3DP), for providing their data. Amitava Bhattacharjee would like to acknowledge the support of DoE grant DEFG02-05ER54832 and NASA grant NNX06AC19G. Ben Chandran acknowledges the support of the NSF/DOE Partnership in Basic Plasma Science and Engineering under grant AST-0613622 and by NASA under grants NNX07AP65G and NNX08AH52G. The data were downloaded via the Internet from the Space Physics Data Facility http://cdaweb.gsfc.nasa. gov/at NASA Goddard Space Flight Center.

[32] Steven Cranmer thanks Peter Goldreich and Sean Oughton for their assistance in evaluating this paper.

\section{References}

Barnes, A. (1983), Hydromagnetic waves, turbulence, and collisionless processes in the interplanetary medium, in Solar-Terrestrial Physics. Principles and Theoretical Foundations, edited by R. L. Carovillano and J. M. Forbes, p. 155, D. Reidel, Dordrecht, Netherlands.

Beresnyak, A., and A. Lazarian (2006), Polarization intermittency and its influence on MHD turbulence, Astrophys. J., 640, L175.

Beresnyak, A., and A. Lazarian (2007), Strong imbalanced turbulence, Astrophys. J., 682, 1070-1075, doi:10.1086/589428.

Bhattacharjee, A., and C. S. Ng (2001), Random scattering and anisotropic turbulence of shear Alfvén wave packets, Astrophys. J., 548, 318

Bieber, J. W., W. H. Matthaeus, C. W. Smith, W. Wanner, M.-B. Kallenrode, and G. Wibberenz (1994), Proton and electron mean free paths: The Palmer consensus revisited, Astrophys. J., 420, 294.

Bieber, J. W., W. Wanner, and W. H. Matthaeus (1996), Dominant twodimensional solar wind turbulence with implications for cosmic ray transport, J. Geophys. Res., 101, 2511.

Boldyrev, S. (2005), On the spectrum of magnetohydrodynamic turbulence, Astrophys. J., 626, L37.

Boldyrev, S. (2006), Spectrum of magnetohydrodynamic turbulence, Phys. Rev. Lett., 96, 115002, doi:10.1103/PhysRevLett.96.115002.

Bruno, R., and V. Carbone (2005), The solar wind as a turbulence laboratory, Living Rev. Sol. Phys., 2(4), (Available at http://www.livingreviews. org/lrsp-2005-4). 
Chandran, B. D. G. (2005), Weak compressible magnetohydrodynamic turbulence in the solar corona, Phys. Rev. Lett., 95, 265004, doi:10.1103/ PhysRevLett.95.265004.

Chandran, B. D. G. (2008a), Strong anisotropic MHD turbulence with cross helicity, Astrophys. J., 685, 646.

Chandran, B. D. G. (2008b), Weakly turbulent magnetohydrodynamic waves in compressible low- $\beta$ plasmas, Phys. Rev. Lett., 101, 235004 doi:10.1103/PhysRevLett.101.235004.

Chen, S. Y., and R. H. Kraichnan (1997), Inhibition of turbulent cascade by sweep, J. Plasma Phys., 57, 187.

Cho, J., and A. Lazarian (2003), Compressible magnetohydrodynamic turbulence: Mode coupling, scaling relations, anisotropy, viscosity-damped regime and astrophysical implications, Mon. Not. R. Astron. Soc., 345 , 325 .

Cho, J., and E. T. Vishniac (2000), The anisotropy of magnetohydrodynamic Alfvénic turbulence, Astrophys. J., 539, 273.

Dasso, S., L. J. Milano, W. H. Matthaeus, and C. W. Smith (2005), Anisotropy in fast and slow solar wind fluctuations, Astrophys. J., 635, L181.

Galtier, S., S. V. Nazarenko, A. C. Newell, and A. Pouquet (2002), Anisotropic turbulence of shear-Alfvén waves, Astrophys. J., 564, L49.

Galtier, S., A. Pouquet, and A. Mangeney (2005), On spectral scaling laws for incompressible, anisotropic magnetohydrodynamic turbulence, Phys. Plasmas, 12, 092310, doi:10.1063/1.2052507.

Goldreich, P., and S. Sridhar (1995), Toward a theory of interstellar turbulence II: Strong Alfvénic turbulence, Astrophys. J., 438, 763.

Goldreich, P., and S. Sridhar (1997), Magnetohydrodynamic turbulence revisited, Astrophys. J., 485, 680.

Goldstein, B. E., E. J. Smith, A. Balogh, T. S. Horbury, M. L. Goldstein, and D. A. Roberts (1995), Properties of magnetohydrodynamic turbulence in the solar wind as observed by Ulysses at high heliographic latitudes, Geophys. Res. Lett., 22, 3393.

Goldstein, M. L., D. A. Roberts, and W. A. Matthaeus (1995), Magnetohydrodynamic turbulence in the solar wind, Annu. Rev. Astron. Astrophys. $33,283$.

Grappin, R., J. Leorat, and A. Pouquet (1983), Dependence of MHD turbulence spectra on the velocity field-magnetic field correlation, Astron. Astrophys., 126, 51.

Howes, G. G., S. C. Cowley, W. Dorland, G. W. Hammett, E. Quataert, and A. A. Schekochihin (2008), A model of turbulence in magnetized plasmas: Implications for the dissipation range in the solar wind, J. Geophys Res., 113, A05103, doi:10.1029/2007JA012665.

Iroshnikov, P. S. (1964), Turbulence of a conducting fluid in a strong magnetic field, Sov. Astron., 7, 566.

Kraichnan, R. H. (1965), Inertial range spectrum of hydromagnetic turbulence, Phys. Fluids, 8, 1385.

Lepping, R. P., et al. (1995), The WIND magnetic field investigation, Space Sci. Rev., 71, 207.

Lin, R. P., et al. (1995), A three-dimensional plasma and energetic particle investigation for the Wind spacecraft, Space Sci. Rev., 71, 125.

Lithwick, Y., P. Goldreich, and S. Sridhar (2007), Imbalanced strong MHD turbulence, Astrophys. J., 655, 269.

Maron, J., and P. Goldreich (2001), Simulations of incompressible magnetohydrodynamic turbulence, Astrophys. J., 554, 1175.

Marsch, E. (1991), MHD turbulence in the solar wind, in Physics of the Inner Heliosphere II, edited by R. Schwenn and E. Marsch, p. 159 , Springer, Berlin.

Marsch, E., and C.-Y. Tu (1990), On the radial evolution of MHD turbulence in the inner heliosphere, J. Geophys. Res., 95, 8211.

Mason, J., F. Cattaneo, and S. Boldyrev (2006), Dynamic alignment in driven magnetohydrodynamic turbulence, Phys. Rev. Lett., 97, 255002, doi:10.1103/PhysRevLett.97.255002.

Mason, J., F. Cattaneo, and S. Boldyrev (2008), Numerical measurements of the spectrum in magnetohydrodynamic turbulence, Phys. Rev. E, 77, 036403, doi:10.1103/PhysRevE.77.036403.

Matthaeus, W. H., and M. L. Goldstein (1982), Measurement of the rugged invariants of magnetohydrodynamic turbulence in the solar wind, J. Geophys. Res., 87, 6011.

Matthaeus, W. H., A. Pouquet, P. D. Mininni, P. Dmitruk, and B. Breech (2008), Rapid alignment of velocity and magnetic field in magnetohy- drodynamic turbulence, Phys. Rev. Lett., 100, 085003, doi:10.1103/ PhysRevLett.100.085003.

Milano, L. J., S. Dasso, W. H. Matthaeus, and C. W. Smith (2004), Spectral distribution of the cross helicity in the solar wind, Phys. Rev. Lett., 93, 155005, doi:10.1103/PhysRevLett.93.155005.

Mininni, P. D., and A. Pouquet (2007), Energy spectra stemming from interactions of Alfvén waves and turbulent eddies, Phys. Rev. Lett., 99, 254502, doi:10.1103/PhysRevLett.99.254502.

Montgomery, D., and W. H. Matthaeus (1995), Anisotropic modal energy transfer in interstellar turbulence, Astrophys. J., 447, 706.

Montgomery, D., and L. Turner (1981), Anisotropic magnetohydrodynamic turbulence in a strong external magnetic field, Physics of Fluids, 24, $825-831$.

Müller, W.-C., D. Biskamp, and R. Grappin (2003), Statistical anisotropy of magnetohydrodynamic turbulence, Phys. Rev. E, 67, 066302, doi:10.1103/ PhysRevE.67.066302.

$\mathrm{Ng}, \mathrm{C}$. S., and A. Bhattacharjee (1996), Interaction of shear-Alfvén wave packets: Implication for weak magnetohydrodynamic turbulence in astrophysical plasmas, Astrophys. J., 465, 845.

$\mathrm{Ng}, \mathrm{C}$. S., A. Bhattacharjee, K. Germaschewski, and S. Galtier (2003), Anisotropic fluid turbulence in the interstellar medium and the solar wind, Phys. Plasmas, 10, 1954

Oughton, S., W. H. Matthaeus, and S. Ghosh (1995), Anisotropy in incompressible and compressible 3D MHD turbulence, in Small-Scale Structures in Three-Dimensional Hydrodynamic and Magnetohydrodynamic Turbulence, Lect. Notes Phys., vol. 462, p. 273, Springer, Berlin.

Podesta, J. J., D. A. Roberts, and M. L. Goldstein (2006), Power spectrum of small-scale turbulent velocity fluctuations in the solar wind, J. Geophys. Res., 111, A10109, doi:10.1029/2006JA011834.

Podesta, J. J., D. A. Roberts, and M. L. Goldstein (2007), Spectral exponents of kinetic and magnetic energy spectra in solar wind turbulence, Astrophys. J., 664, 543-548.

Podesta, J. J., A. Bhattacharjee, B. D. G. Chandran, M. L. Goldstein, and D. A. Roberts (2008), Scale dependent alignment between velocity and magnetic field fluctuations in the solar wind and comparisons to Boldyrev's phenomenological theory, in Particle Acceleration and Transport in the Heliosphere and Beyond, AIP Conf. Proc., 1039 81-86, doi:10.1063/1.2982489.

Roberts, D. A., M. L. Goldstein, L. W. Klein, and W. H. Matthaeus (1987a), The nature and evolution of magnetohydrodynamic fluctuations in the solar wind: Voyager observations, J. Geophys. Res., 92, 11,021.

Roberts, D. A., M. L. Goldstein, L. W. Klein, and W. H. Matthaeus (1987b), Origin and evolution of fluctuations in the solar wind: HELIOS observations and Helios-Voyager comparisons, J. Geophys. Res., 92, 12,023 .

Shebalin, J. V., W. H. Matthaeus, and D. Montgomery (1983), Anisotropy in MHD turbulence due to a mean magnetic-field, J. Plasma Phys., 29, 525 .

Smith, C. W. (2003), The geometry of turbulent magnetic fluctuations at high heliograpahic latitudes, in Solar Wind Ten: Proceedings of the Tenth International Solar Wind Conference, AIP Conf. Proc., 679, 413.

Sridhar, S., and P. Goldreich (1994), Toward a theory of interstellar turbulence. I. Weak Alfvénic turbulence, Astrophys. J., 432, 612.

Terry, P. W., C. Mackay, and E. Fernandez (2001), The role of electron density in magnetic turbulence, Phys. Plasmas, 8, 2707.

Tu, C.-Y., E. Marsch, and K. M. Thieme (1989), Basic properties of solar wind MHD turbulence near $0.3 \mathrm{AU}$ analyzed by means of Elsässer variables, J. Geophys. Res., 94, 11,739.

Tu, C.-Y., and E. Marsch (1995), MHD structures, waves and turbulence in the solar wind: Observations and theories, Space Sci. Rev., 73, 1.

A. Bhattacharjee, B. D. G. Chandran, and J. J. Podesta, Space Science Center, University of New Hampshire, Durham, NH 03824, USA. (jpodesta@solar.stanford.edu)

M. L. Goldstein and D. A. Roberts, Laboratory for Geospace Physics, NASA Goddard Space Flight Center, Code 673, Greenbelt, MD 20771, USA. 